\title{
ERRATUM
}

Hans-Rudolf Aerni · Bernd Kobler

Barbara V. Rutishauser · Felix E. Wettstein · René Fischer

Walter Giger · Andreas Hungerbühler

M. Dolores Marazuela $\cdot$ Armin Peter · René Schönenberger

A. Christiane Vögeli · Marc J.-F. Suter · Rik I. L. Eggen

\section{Combined biological and chemical assessment of estrogenic activities in wastewater treatment plant effluents}

Published online: 26 February 2004

(c) Springer-Verlag 2004

\section{Anal Bioanal Chem (2004) 378:688-696}

Hans-Rudolf Aerni, Bernd Kobler, Barbara V. Rutishauser and Felix E. Wettstein contributed equally and should be considered as first authors.

The online version of the original article can be found at http://dx.doi.org/10.1007/s00216-003-2276-4

H.-R. Aerni · B. Kobler · B. V. Rutishauser · F. E. Wettstein

W. Giger · A. Hungerbühler · A. Peter · R. Schönenberger

A. C. Vögeli · M. J.-F. Suter (凶) · R. I. L. Eggen

Swiss Federal Institute for Environmental Science

and Technology (EAWAG),

Ueberlandstrasse 133, P.O. Box 611, 8600 Dubendorf, Switzerland

e-mail: marc.suter@eawag.ch

R. Fischer

Swiss Federal Institute of Technology (ETH),

8092 Zurich, Switzerland

M. D. Marazuela

Complutense University, 28040 Madrid, Spain 